\title{
COMUNICACÕES
}

\section{Ocurrencia de Bipolaris sorokiniana en plantas de soja en Argentina}

\author{
María Graciela Cabrera ${ }^{1}$, María Agueda Cúndom ${ }^{1}$, Álvarez Roberto Eloy ${ }^{1}$, Susana Alejandra Gutiérrez ${ }^{1}$
}

${ }^{1}$ Cátedra de Fitopatología, Facultad de Ciencias Agrarias, UNNE. Sargento Cabral 2131 (3400) Corrientes, Argentina. e-mail: cabrera@ agr.unne.edu.ar Autor para correspondência: Susana Alejandra Gutiérrez (cabrera@agr.unne.edu.ar)

Data de chegada: 22/01/2009. Aceito para publicação em: 10/01/2010.

La soja (Glycine max (L.) Merr.), cubre la mayor superficie sembrada con una especie de planta en Argentina y por lo tanto, es el cultivo que sufre los mayores ataques de enfermedades y plagas respecto a los restantes cultivos del país. Varios hongos causan lesiones sobre los distintos órganos de la parte aérea de soja, causando síntomas necróticos (Cabrera et al. Hongos menores detectados en follaje de soja en Chaco y Corrientes, Argentina. 2006. Disponible en http://www.unne.edu.ar /Web/cyt/cyt2006/index.htm; Distéfano de Vallone, S.; Giorda, L. Editores Enfermedades de la soja en la Argentina. INTA Centro Regional Córdoba, 1997. 72 p.).

Durante las campañas 2002 al 2008 se observaron síntomas necróticos en hojas, vainas y semillas de plantas de soja procedentes de las provincias de Chaco, Corrientes y Formosa (Argentina), que se asociaron a un hongo con características morfológicas del género Bipolaris Shoemaker. Los antecedentes bibliográficos consultados mencionan a Helminthosporium vignae L. S. (Index of Plant Diseases in the United States, Agriculture Handbook $\mathrm{N}^{\circ} 165,1960,531 \mathrm{p}$.), causando manchas en hojas, vainas y semillas de soja y Drechslera glycini Narayanasamy y Durairaj, ocasionando tizón foliar y muerte de plántulas (Hartman, G. L. et al. Compendium of Soybean Diseases, 4th. ed. St. Paul: The American Phytopathological Society, 1999. 100 p.)

El objetivo del trabajo fue conocer la nueva enfermedad presente en plantas de soja procedentes de cultivos del nordeste argentino e identificar al agente causal.

Se examinaron 30 muestras de plantas de soja en diferentes lotes de cultivo de las provincias de Corrientes, Chaco y Formosa (Argentina) durante la campaña 2007/08, con microscopio estereoscópico ( $35 \mathrm{x})$, seleccionándose todas las hojas con síntomas de enfermedad, las que fueron incubadas en cámaras húmedas a

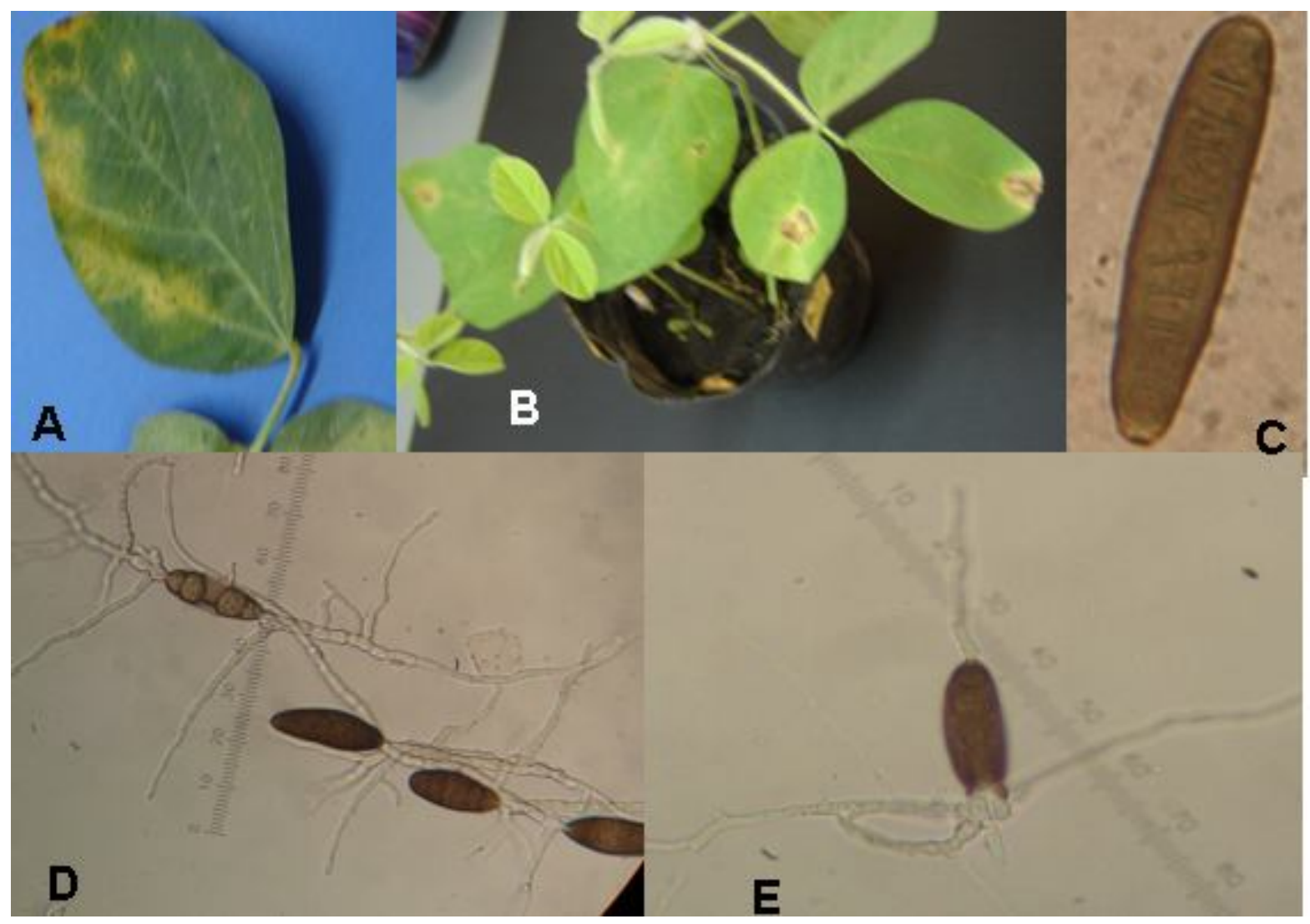

Figura 1. A. Síntomas de tizón causados por Bipolaris sorokiniana originados en infección natural de soja. B. Detalle de infección por inoculación en planta sana. C. Conidio originado en infección natural de soja. D y E. Germinación de conidios originados en medio de cultivo. 
temperatura ambiente. Para determinar la morfología del organismo asociado a los síntomas, se examinaron con microscopio óptico (400 $\mathrm{x})$, preparados montados en agua destilada estéril. Se efectuaron aislamientos en medio agar papa glucosa (APG) al 1,5\%, pH 6,5, en condiciones de $12 \mathrm{~h} \mathrm{luz}, 12 \mathrm{~h}$ oscuridad, y temperatura de $25-27^{\circ} \mathrm{C}$. La patogenicidad del agente causal se determinó mediante inoculaciones en plantas sanas de soja, criadas en macetas en invernáculo. Discos de medio de cultivo conteniendo inóculo (micelio y conidios) se colocaron individualmente sobre hojas y ramas de plantas de soja.

En hojas de plantas enfermas de soja, se observaron lesiones irregulares, extensivas, de color verde oliváceo, inicialmente de aspecto húmedo, con márgenes de color castaño claro, rodeadas de un halo clorótico. El tizón causó un severo efecto destructivo en plantas muy jóvenes. Las inoculaciones experimentales reprodujeron los síntomas de la enfermedad a partir de las 48 horas, con desarrollo extensivo de lesiones húmedas; de dichas lesiones se reaisló el mismo hongo (Fig.1.B).

En APG, el hongo patógeno desarrolló colonias compactas, densas, de color gris oliváceo a negro y reverso oscuro casi negro. Desde el micelio desarrollaron conidióforos libres, simples, geniculados, septados, marrón claro, con cicatrices conidiales evidentes. Tanto sobre las lesiones de infecciones naturales como en los aislamientos se produjeron abundantes conidios fusiformes, de color castaño oscuro con extremos más claros, con cicatriz hilar visible, con 4-9-12 pseudoseptos y tamaño variable; midieron entre $24-119 \mu \mathrm{m} \times 13-28 \mu \mathrm{m}$. Germinaron en pocas horas, en ambiente saturado de humedad, produciendo tubos germinativos generalmente por ambos extremos; algunos conidios germinaron por un solo extremo, desarrollando un tubo simple, largo, sinuoso, tabicado y geniculado (Fig.1. C, D).

Las características morfométricas coincidieron con las señaladas por Ellis M. B. (More Dematiaceous Hyphomycetes. Commonwealth Mycological Institute, Kew, UK, 1996. 507 p.), y Sivanesan, A. (Graminicolous Species of Bipolaris, Curvularia, Drechslera, Exerohilum and Their Teleomorphs, Mycological Papers $\mathrm{N}^{\circ}$ 158, C.A.B., Internacional Mycological Institute, 1987. 261 p.) para Bipolaris sorokiniana (Sacc. ex Sorok.) Shoemaker.

Por el momento los conocimientos sobre la enfermedad son insuficientes para establecer el nivel de daño que podría causar en los cultivos de la región nordeste de Argentina, siendo necesario continuar con éste tipo de estudios para establecerlos. También se debe mencionar la importancia que representa el patógeno como causante de la mancha borrosa o marrón del trigo y cebada, el cual produce lesiones en coleóptiles, plúmulas, hojas y vainas (Wiese, M.V. Compendium of wheat disease. 2nd. ed. St. Paul: The American Phytopathological Society, 1987. 106 p.). En Argentina, en las últimas campañas agrícolas se detectó un aumento en la incidencia de este patógeno en cultivos y semillas de trigo, principalmente en regiones cálidas, como las que se presentan en la región nordeste del país (Carmona, M. ed. Manual para el Manejo Integrado de Enfermedades en Trigo. 2ed., 2005. 62 p.). Constituye esta la primera cita de $B$. sorokiniana como agente causal de tizón en plantas de soja en Argentina. 